\title{
Practice variation in anti-epileptic drug use for neonatal hypoxic-ischemic encephalopathy among regional NICUs
}

\author{
Maria L. V. Dizon ${ }^{1 *}$ (D), Rakesh Rao², Shannon E. Hamrick ${ }^{3}$, Isabella Zaniletti ${ }^{4}$, Robert DiGeronimo ${ }^{5}$, Girija Natarajan ${ }^{6}$, \\ Jeffrey R. Kaiser ${ }^{7}$, John Flibotte ${ }^{8}$, Kyong-Soon Lee ${ }^{9}$, Danielle Smith ${ }^{10}$, Toby Yanowitz ${ }^{11}$, Amit M. Mathur ${ }^{2}$ and \\ An N. Massaro ${ }^{12}$
}

\begin{abstract}
Background: While intercenter variation (ICV) in anti-epileptic drug (AED) use in neonates with seizures has been previously reported, variation in AED practices across regional NICUs has not been specifically and systematically evaluated. This is important as these centers typically have multidisciplinary neonatal neurocritical care teams and protocolized approaches to treating conditions such as hypoxic ischemic encephalopathy (HIE), a population at high risk for neonatal seizures. To identify opportunities for quality improvement (QI), we evaluated ICV in AED utilization for neonates with HIE treated with therapeutic hypothermia (TH) across regional NICUs in the US.
\end{abstract}

Methods: Children's Hospital Neonatal Database and Pediatric Health Information Systems data were linked for 1658 neonates $\geq 36$ weeks' gestation, > $1800 \mathrm{~g}$ birthweight, with HIE treated with TH, from 20 NICUs, between 2010 and 2016. ICV in AED use was evaluated using a mixed-effect regression model. Rates of AED exposure, duration, prescription at discharge and standardized AED costs per patient were calculated as different measures of utilization.

Results: Ninety-five percent (range: $83-100 \%)$ of patients with electrographic seizures, and 26\% (0-81\%) without electrographic seizures, received AEDs. Phenobarbital was most frequently used (97.6\%), followed by levetiracetam (16.9\%), phenytoin/fosphenytoin (15.6\%) and others (2.4\%; oxcarbazepine, topiramate and valproate). There was significant ICV in all measures of AED utilization. Median cost of AEDs per patient was $\$ 89.90$ (IQR $\$ 24.52, \$ 258.58$ ).

Conclusions: Amongst Children's Hospitals, there is marked ICV in AED utilization for neonatal HIE. Variation was particularly notable for HIE patients without electrographic seizures, indicating that this population may be an appropriate target for QI processes to harmonize neuromonitoring and AED practices across centers.

Keywords: Hypoxic-ischemic encephalopathy, Anti-epileptic drugs, Neonatal seizures

\section{Background}

Although seizures occur in $26-65 \%$ of neonates with hypoxic-ischemic encephalopathy (HIE), it is well known that anti-epileptic drug (AED) management is variable among centers [1-4]. There are several possible reasons for this variability. Neonatal seizures are often subclinical, difficult to detect and cannot be predicted adequately by clinical variables alone [5, 6]. Furthermore,

\footnotetext{
* Correspondence: m-dizon@northwestern.edu

${ }^{1}$ Ann \& Robert H. Lurie Children's Hospital of Chicago and Feinberg School of Medicine, Northwestern University, 225 East Chicago Ave, Box 45, Chicago, IL 60611, USA

Full list of author information is available at the end of the article
}

limitations in available resources to detect seizures, as well as a lack of consensus for seizure management among treating neonatologists and child neurologists lead to inconsistent recognition and treatment of neonatal seizures $[7,8]$. Continuous electroencephalographic (cEEG) monitoring is therefore recommended in the management of neonates with encephalopathy [9]. However, cEEG is resource intensive and may not be available in all cooling centers. Even when available, factors such as time to application and interpretation may not be uniform across centers. Amplitude-integrated EEG (aEEG) is an alternative form of easily interpretable neuromonitoring that is routinely used in many but not

(c) The Author(s). 2019 Open Access This article is distributed under the terms of the Creative Commons Attribution 4.0 International License (http://creativecommons.org/licenses/by/4.0/), which permits unrestricted use, distribution, and reproduction in any medium, provided you give appropriate credit to the original author(s) and the source, provide a link to the Creative Commons license, and indicate if changes were made. The Creative Commons Public Domain Dedication waiver (http://creativecommons.org/publicdomain/zero/1.0/) applies to the data made available in this article, unless otherwise stated. 
all centers. Finally, the use of selective head cooling for treatment of HIE may temporarily preclude continuous EEG monitoring during therapeutic hypothermia (TH). Detection of subclinical seizures is important because treatment of subclinical seizures reduces seizure burden, and longer duration of seizures is associated with more severe brain injury on MRI and lower performance scores in all domains of the Bayley Scares of Infant Development-III [10, 11].

Variation also exists in the choice of AEDs. Phenobarbital is the first-line AED for treatment of neonatal seizures despite limited evidence to support its use over other agents, [12-14] either for treatment or for seizure prophylaxis [15-17]. Common second-line AEDs for persistent seizures include phenytoin (with similar effectiveness as phenobarbital) [14] and benzodiazepines. More recently, levetiracetam and topiramate are increasingly being used in NICUs as second-line AEDs $[8,18]$ and are under investigation for potential neuroprotective qualities [19]. Lidocaine has also been described as an AED [14, 20]. Unfortunately, the field has few randomized trials in neonates proving safety or efficacy of one AED over another. A clinical trial of bumetanide as a second-line AED for electrographic seizures not responsive to phenobarbital did not show efficacy but did show the serious side-effect of hearing impairment [21]. The recently completed clinical trial of levetiracetam as first-line therapy for neonatal seizures (NEOLEV2 NCT01720667) reportedly did not show greater efficacy of levetiracetam over phenobarbital (Child Neurology Society Annual Meeting, Chicago, IL, October 16, 2018). Consistent and rational use of these drugs is important as pre-clinical and clinical studies have raised concern regarding AED-associated neurotoxicity in the developing brain, with detrimental effects on neurogenesis, cell proliferation and migration, apoptosis, synaptogenesis and white matter integrity [22-26].

Reducing intercenter variation (ICV) through standardization of care has been demonstrated to improve outcomes across NICU populations [27]. Importantly, several centers have shown that protocol-driven management of neonates at risk for seizures results in improvements in care including diagnosis of seizures [28], decreased phenobarbital levels, progression to status epilepticus, length of hospital stay [29] and discharge on AED [30]. (Improvement in outcomes due to protocolized approaches has been shown in management of other neonatal diseases as well, including congenital diaphragmatic hernia [31] and short bowel syndrome [32]). An important step to improving consistency of care is to understand determinants of variability in AED prescribing practices. Recent studies have reported exposure trends over time and ICV in AED use for neonatal seizures $[7,8,33,34]$. A consistent message from these reports is the widespread ICV in AED practices, which is not surprising given that prior investigations have evaluated populations of mixed diagnoses and data from various NICUs with different levels of care. Even though neuromonitoring and neuroimaging technology and child neurology specialists are readily available, CHND NICUs do not share standardized treatment protocols. Therefore, we hypothesized that seizure treatment for HIE would vary among the quaternary care Children's Hospitals in our large consortium. Our objective was to identify sources of ICV in AED utilization with the plan to identify opportunities for quality improvement (QI).

\section{Methods}

Using linked data from the Children's Hospital Neonatal Database (CHND) and Pediatric Health Information Systems (PHIS) we quantified ICV in the use of AEDs (initiation, selection and duration) and AED cost as another proxy measurement of AED use for neonates with HIE.

\section{Data sources}

CHND prospectively captures detailed clinical data from all infants admitted to 34 participating level IV NICUs [27]. PHIS contains detailed hospital administrative and billing data from $>40$ pediatric institutions [35]. Twenty-four CHND sites participate in PHIS. Methods insuring data quality for both databases have been reported [27, 35-38]. CHND and PHIS data were linked at the patient level using unique identifiers unavailable to investigators.

\section{Study population}

CHND was queried to identify neonates born at participating centers between July 2010 and July 2016 with the diagnosis of perinatal HIE according to established criteria [3], treatment with $\mathrm{TH}$, admitted $<2 \mathrm{~d}$ of life, $\geq 36$ weeks' gestation and $\geq 1800 \mathrm{~g}$ at birth. Neonates were excluded if they had major congenital anomalies or if linkage to PHIS was not possible. The Institutional Review Board at each participating institution approved participation in CHND and associated research studies.

\section{Data collection}

Data regarding antenatal, maternal, birth and delivery characteristics including mode of delivery as well as clinical and demographic data were abstracted according to a CHND manual of operations [27]. Additional detailed neurological data were recorded for neonates with HIE including results of continuous electroencephalographic monitoring (cEEG) within $24 \mathrm{~h}$, amplitude-integrated EEG (aEEG) studies at $24 \mathrm{~h}$ and neuroimaging findings on magnetic resonance imaging (MRI). Clinical seizures were also recorded. 


\section{Estimation of AED use}

AED use was quantified using four distinct approaches to capture different aspects of use. PHIS Clinical Transaction Classification (CTC) pharmacy codes corresponding to any type of AED were used to quantify frequency, type and duration of AED use per patient. AED exposure was defined as $\geq 1 \mathrm{AED}$ CTC code during the initial hospitalization in a given patient. AED duration was defined as total number of hospital days with $\geq 1$ AED CTC codes assigned. Neonates were also classified by whether or not they received an $A E D$ at discharge.

\section{Cost estimation}

Standardized costs were calculated according to a previously described cost master index [35, 39]. Briefly, costs for every billing CTC code were computed and adjusted for wage and price index. All costs were inflated to 2012 dollars and a standardized unit cost for each CTC code was defined as the median cost across all participating hospitals. Standardized costs for all AED-associated CTC codes were calculated per patient. Costs were also calculated for individual AEDs (i.e., phenobarbital, levetiracetam, fosphenytoin/phenytoin, oxcarbazeine, topiramate and valproate). Benzodiazepines (midazolam and lorazepam) were not included in overall models because we could not confirm whether these medications were being used as AEDs or for sedation.

\section{Data analysis}

Study sample size was based on a convenience sample of consecutive admissions of infants meeting inclusion criteria during the study period. Study population characteristics and cost distribution data were described using standard summary statistics after stratifying by presence of seizures noted on EEG (cEEG or aEEG). ICV in AED exposure was evaluated using a logistic regression model, ICV in AED duration was evaluated using a generalized linear model and ICV in AED costs per case was evaluated using a mixed-linear model adjusting for gestational age, sex, electrographic seizures during hospitalization, HIE severity and mortality. Cost data were $\log$ transformed to account for the skewed distribution. Data were analyzed using SAS Enterprise Guide 7.1 (SAS Institute Inc., Cary, NC).

\section{Results}

\section{Study population}

Of the 120,601 infants included in the CHND at the time of analysis, we identified 2903 neonates with HIE treated with TH. Neonates admitted at $>2 \mathrm{~d}$ of life, $<36$ weeks' gestation, birthweight $<1800 \mathrm{~g}$, with event timing classified as non-perinatal or with major congenital anomalies were excluded $(n=727)$, leaving 2176 neonates. We were able to link 1744 of the $2176(80 \%)$ remaining neonates to their PHIS data. After eliminating additional neonates with systematic errors in PHIS data, we were left with 1658 of 2176 (76\%) who met study inclusion criteria (Fig. 1). These neonates were cared for at 20 centers in the US. Median beds per NICU at these centers was 60 (range 28-173). The median number of babies treated with $\mathrm{TH}$ per center for the study period was 75 (range 12-187). Each of the centers had a NeuroNICU program and/or the daily involvement of a neurologist. None of the centers provided prophylactic phenobarbital as part of usual practice.

Characteristics of the study population were stratified by presence of clinical or electrographic seizures (Table 1). Ninety-eight percent of all neonates received some form of EEG monitoring (aEEG or cEEG). The exact timing of initiation of cEEG or aEEG monitoring was not known although it was known if studies were done before or after $3 \mathrm{~d}$ of life; almost all studies were done by $24 \mathrm{~h}$ of life. As expected, Apgar scores $\leq 5$ at 5 , 10 and $15 \mathrm{~min}$ of life, encephalopathy severity, resuscitation in the delivery room (including mechanical ventilation, chest compressions and epinephrine), severe acidosis $(\mathrm{pH} \leq 7.00)$ and use of vasopressors were more frequent in neonates with electrographic seizures. There was no difference in acute perinatal sentinel events [40] between groups with the exception of fetal distress. Encephalopathy grade differed by seizure group. The rate of babies with mild-moderate encephalopathy increased from $60 \%$ to over $80 \%$ during the study period. Eighteen percent of the cohort (308 of 1658) had mild encephalopathy; of these $5.8 \%$ had clinical seizures. The majority underwent total body cooling. We observed a higher rate of seizures in neonates who were selectively head-cooled in contrast with those who received whole body cooling. There was no difference between groups in use of inhaled nitric oxide or extracorporeal membrane oxygenation. Unadjusted rates of mortality were higher and lengths of CHND hospital stay were longer in neonates with electrographic seizures (Table 1). Length of hospital stay included total stay in the CHND hospital (i.e. includes within hospital transfer out of the NICU but not to an outside facility for subacute care).

Four hundred seventy-two of 1658 (28.5\%) neonates included in the study had seizures noted on cEEG or aEEG at anytime during the first $24 \mathrm{~h}$ of admission; cEEGs were used in over two-thirds (1131/1658) and aEEG in nearly one-third (494/1658) of neonates. Not surprisingly, the rate of cEEG monitoring was lower in the selectively head-cooled neonates (only 23\% received cEEG within the first $24 \mathrm{~h}$ of admission compared to $81 \%$ for whole body-cooled neonates; $47 \%$ of head-cooled neonates received aEEG compared to $27 \%$ for whole body-cooled). Status epilepticus was noted in $2 \%$ of all patients $(n=27)$ or $6 \%$ of patients with 


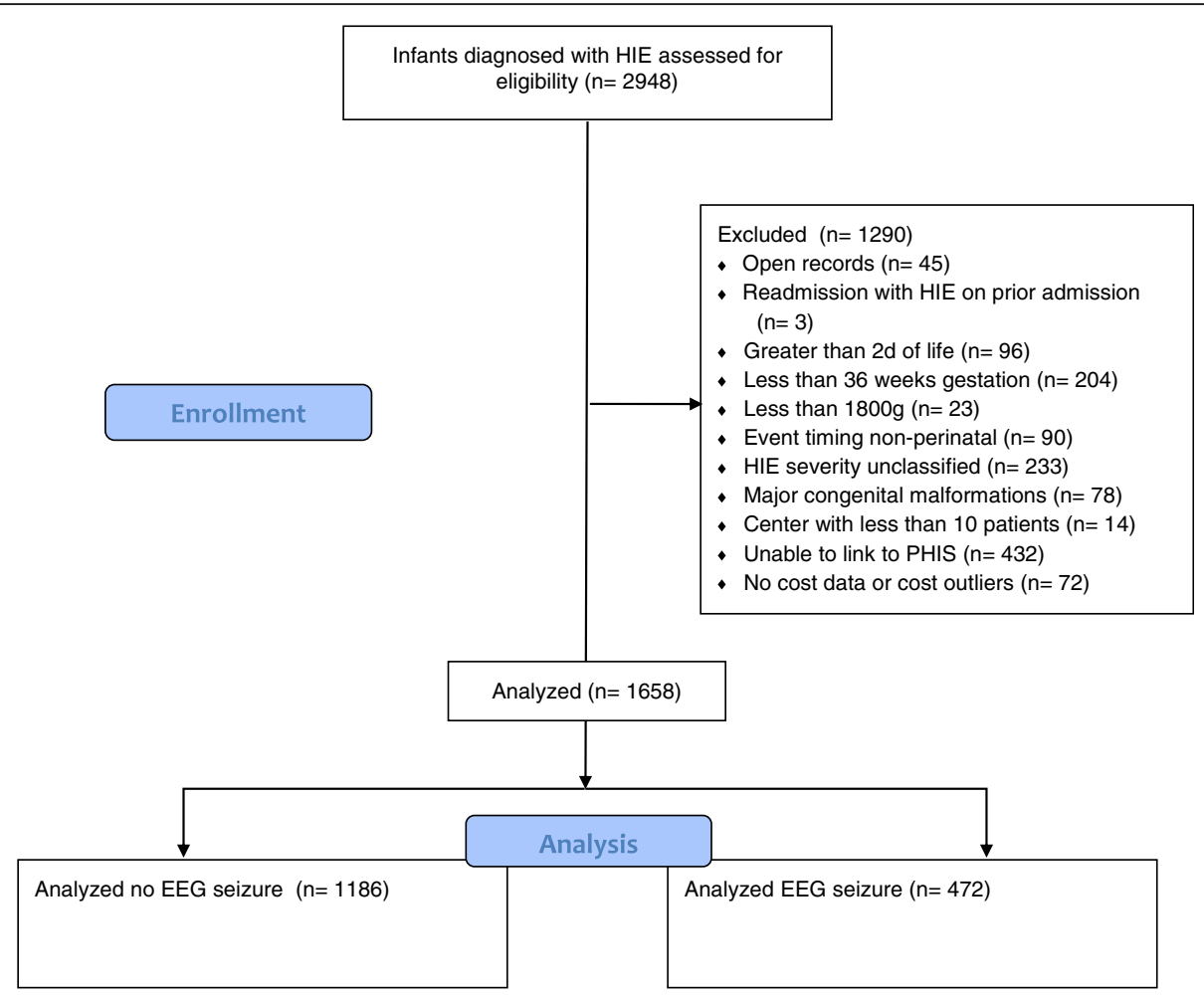

Fig. 1 CONSORT flow diagram

electrographic seizures. Neonates with seizures on cEEG were more likely to have an abnormal background reported at $24 \mathrm{~h}$ (Table 2). In contrast, clinical seizures that were not present electrographically were observed in 239 of $1186(20 \%)$ neonates (Table 1). Of these, 200 occurred at or before $3 \mathrm{~d}$ of life and 39 occurred after $3 \mathrm{~d}$ of life. Interestingly, $5.8 \%$ of cases of mild encephalopathy had clinical seizures (1.1\% of the entire cohort) and 9.4\% had EEG seizures (1.8\% of the entire cohort). On neuroimaging, MRI was completed in 1450 (87\%) of neonates and was more often abnormal in neonates with electrographic seizures, with a higher incidence of hemorrhage, stroke, white matter injury and injury to cortex or deep grey nuclei (Table 2).

\section{AED selection}

Among patients receiving AEDs with the exclusion of midazolam, lorazepam and clonazepam $(n=757)$, phenobarbital was used most frequently (97.6\%), followed by levetiracetam (16.9\%), fosphenytoin/phenytoin $(15.6 \%)$ and others (2.5\%; oxcarbazepine, topiramate, valproate) (Table 3 ). Unadjusted ICV in patient exposure to phenobarbital (Fig. 2b), levetiracetam and phenytoin/fosphenytoin (Fig. 2c) across 20 centers was striking. Frequency of exposure to levetiracetam and fosphenytoin/phenytoin appeared inversely related to each other by center. Two hundred and ninety-five
(39\%) of patients received only 1 AED, whereas 250 (33\%) received 2 and 212 (29\%) received 3 or more AEDs. Phenobarbital was the first-line AED throughout the entire study period. The most common second drug changed at the end of the study from fosphenytoin/ phenytoin to levetiracetam (Fig. 4g). Interestingly, 10 patients received levetiracetam only. Of note, benzodiazepines were given to $95 \%$ of patients.

\section{AED use in neonates with HIE treated with TH}

AEDs were given in $45 \%$ of patients overall. Frequencies of AED exposure stratified by the presence of electrographic seizures are shown in Fig. 2a. In patients with electrographic seizures, AED exposure was nearly universal (95\%, range $83-100 \%$ across centers). Surprisingly, a significant proportion of neonates $(26 \%$, range $0-81 \%$ across centers) who received AED did not have seizures captured on any type of EEG (Fig. 2a), and in only one center (center 11) no neonate without EEG seizures received an AED. In a logistic regression model adjusting for gestational age, sex, electrographic seizures, status epilepticus, HIE severity and mortality, AED exposure differed significantly across centers $(p<0.001)$ (Fig. 3a). The magnitude of adjusted differences between centers for any AED exposure was estimated as high as 15-fold. Likewise, AED duration (Fig. 3b), evaluated with a generalized linear model, also differed significantly across 
Table 1 Clinical characteristics of study subjects

\begin{tabular}{|c|c|c|c|c|c|}
\hline & All & No Seizure & EEG Seizure & Clinical Seizure & $p$-value \\
\hline & 1658 & 947 & 472 & 239 & \\
\hline Gestational age in weeks Mean (SD) & $38.80(1.48)$ & $38.74(1.49)$ & $38.97(1.48)$ & $39(1.41)$ & $0.010^{*}$ \\
\hline Birth weight in grams Median [IQR] & $3290[2940,3700]$ & $3295[2940,3715]$ & $3270[2890,3635]$ & $3310[3000,3720]$ & 0.206 \\
\hline Female sex (n, \%) & $721(43.5)$ & $395(41.7)$ & $223(47.3)$ & $103(43.1)$ & 0.139 \\
\hline Maternal race $(\mathrm{n}, \%)$ & & & & & $0.007^{*}$ \\
\hline White & $996(60.1)$ & $569(60.1)$ & $289(61.2)$ & $138(57.7)$ & \\
\hline Black & $350(21.1)$ & $217(22.9)$ & $92(19.5)$ & $41(17.2)$ & \\
\hline Asian & $72(4.3)$ & $43(4.5)$ & $20(4.2)$ & $9(3.8)$ & \\
\hline Other & $164(9.9)$ & $73(7.7)$ & $51(10.8)$ & $40(16.7)$ & \\
\hline Delivery type $(n, \%)$ & & & & & 0.862 \\
\hline Cesarean & $1069(64.5)$ & $617(65.2)$ & $301(63.8)$ & $151(63.2)$ & \\
\hline Vaginal, non-operative & $407(24.6)$ & $223(23.6)$ & $120(25.4)$ & $64(26.8)$ & \\
\hline Vaginal, operative & 175(10.6) & $102(10.8)$ & 49 (10.4) & $24(10.0)$ & \\
\hline Unknown & $7(0.42)$ & $5(0.53)$ & $2(0.42)$ & $0(0)$ & \\
\hline \multicolumn{6}{|l|}{ Apgar score $<=5$} \\
\hline 5 min Median [IQR] & $3[1,4]$ & $3[2,4]$ & $2[1,4]$ & $2[1,4]$ & $<0.001^{*}$ \\
\hline 10 min Median [IQR] & $3[2,5]$ & $4[2,5]$ & $3[2,4]$ & $3[1,4]$ & $<0.001^{*}$ \\
\hline 15 min Median [IQR] & $3[2,5]$ & $4[2,5]$ & $3[2,4]$ & $2[1,4]$ & $0.012^{*}$ \\
\hline \multicolumn{6}{|l|}{ DR resuscitation } \\
\hline ETTNentilation (n, \%) & $1190(71.8)$ & $631(66.6)$ & $375(79.5)$ & $184(77.0)$ & $<0.001^{*}$ \\
\hline Chest compressions ( $n, \%)$ & $688(41.5)$ & $319(33.7)$ & $238(50.4)$ & $131(54.8)$ & $<0.001^{*}$ \\
\hline Epinephrine (n, \%) & $375(22.6)$ & $156(16.5)$ & $144(30.5)$ & $75(31.4)$ & $<0.001^{*}$ \\
\hline Presenting $\mathrm{pH}^{\#}$ Median [IQR] & $6.99[6.82,7.14]$ & $7.02[6.88,7.15]$ & $6.96[6.80,7.12]$ & $6.9[6.80,7.10]$ & $0.001^{*}$ \\
\hline Presenting BD Median [IQR] & $16[11.5,21]$ & $15[11.5,18.8]$ & $17[12,22.3]$ & $17.9[13.3,24.8]$ & $0.001^{*}$ \\
\hline \multicolumn{6}{|l|}{ Perinatal sentinel event } \\
\hline Nuchal cord (n, \%) & $288(17.4)$ & $174(18.4)$ & 79 (16.7) & 35 (14.6) & 0.36 \\
\hline Cord prolapse (n, \%) & $54(3.3)$ & $31(3.3)$ & $11(2.3)$ & $12(5.0)$ & 0.16 \\
\hline Uterine rupture (n, \%) & $96(5.8)$ & $46(4.9)$ & $34(7.2)$ & $16(6.7)$ & 0.17 \\
\hline Placental abruption (n, \%) & $196(11.8)$ & $99(10.5)$ & $67(14.2)$ & $30(12.6)$ & 0.11 \\
\hline Fetal distress (n, \%) & $362(21.8)$ & $232(24.5)$ & $91(19.3)$ & $39(16.3)$ & $0.007^{*}$ \\
\hline Continuous inotropes on transport/admission ( $\mathrm{n}, \%)$ & $318(19.2)$ & $150(15.8)$ & $116(24.6)$ & $52(21.8)$ & $<0.001^{*}$ \\
\hline \multicolumn{6}{|l|}{ Maternal antenatal conditions } \\
\hline Chorioamnionitis (n, \%) & $140(8.4)$ & $78(8.24)$ & $49(10.4)$ & $13(5.4)$ & 0.08 \\
\hline Diabetes (n, \%) & $198(11.9)$ & $126(13.3)$ & $36(7.6)$ & $36(15.1)$ & $0.002^{*}$ \\
\hline Encephalopathy severity $^{+}$ & & & & & $<0.001^{*}$ \\
\hline Mild (n, \%) & 308 (18.6) & 261 (27.6) & $29(6.1)$ & $18(7.5)$ & \\
\hline Moderate (n, \%) & $917(55.3)$ & $560(59.1)$ & $227(48.1)$ & $130(54.4)$ & \\
\hline Severe (n, \%) & $433(26.1)$ & $126(13.3)$ & $216(45.8)$ & $91(38.1)$ & \\
\hline Type of cooling & & & & & $<0.001^{*}$ \\
\hline Head (n, \%) & $337(20.3)$ & $121(12.8)$ & $153(32.4)$ & $63(26.4)$ & \\
\hline Whole body $(n, \%)$ & $1326(80.0)$ & $834(88.1)$ & $320(67.8)$ & $172(72.0)$ & \\
\hline ECMO & $57(3.4)$ & $37(3.9)$ & $10(2.1)$ & $10(4.2)$ & 0.17 \\
\hline iNO & $334(20.14)$ & $182(19.2)$ & $90(19.07)$ & $62(25.9)$ & 0.05 \\
\hline Pre-discharge mortality (n, \%) & $223(13.5)$ & $85(9.0)$ & $93(19.7)$ & $45(18.8)$ & $<0.001^{*}$ \\
\hline
\end{tabular}


Table 1 Clinical characteristics of study subjects (Continued)

\begin{tabular}{llllll}
\hline & All & No Seizure & EEG Seizure & Clinical Seizure & $p$-value \\
\hline Survivors hospital LOS Median [IQR] & $13[10,22]$ & $11[8,18]$ & $15[10,25.5]$ & $14[9,26]$ & $<0.001^{*}$ \\
Treated with AED $(n, \%)$ & $757(45.6)$ & $133(11.9)$ & $447(94.7)$ & $197(82.4)$ & $<0.001^{*}$ \\
Discharged on AED $(n, \%)$ & $261(20.1)$ & $11(1.42)$ & $196(56.3)$ & $54(30.7)$ & $<0.001^{*}$ \\
\hline
\end{tabular}

Abbreviations: EEG electroencephalographic, IQR interquartile range, $D R$ delivery room, ETT endotracheal tube, ECMO extracorporeal membrane oxygenation, iNO inhaled nitric oxide, LOS length of stay, AED anti-epileptic drug

\#Presenting $\mathrm{pH}=$ worst umbilical cord gas or arterial blood gas within $1 \mathrm{~h}$ of life if cord gas not available

${ }^{+}$VON or NICHD definitions of HIE were used, depending on each site's practice; for the NICHD definition, infants with mild encephalopathy on Sarnat exam and seizures qualify for $\mathrm{TH}$

${ }^{*} p$-value $<=0.05$

centers after adjusting for gestational age, sex, electrographic seizures, status epilepticus, HIE severity, mortality and length of stay $(p<0.001)$. Days of exposure to AEDs ranged between 0.5 fold to 1.5 -fold adjusted differences. As expected, neonates with electrographic seizures were more frequently discharged on AEDs as compared to those without (56\% vs. 6.9\%) (Table 1 ). After adjustment, results from logistic regression show a significant difference across centers in AED use at discharge $(p<0.001)$, as much as 6 -fold (Fig. 3c).

Table 2 Neurophysiologic and MRI findings of study subjects

\begin{tabular}{|c|c|c|c|c|c|}
\hline & All & No Seizure & EEG Seizure & Clinical Seizure & $p$-value \\
\hline & 1658 & 947 & 472 & 239 & \\
\hline $\mathrm{aEEG}$ at $24 \mathrm{~h}^{\#}(\mathrm{n}, \%)$ & $494(29.8)$ & $259(27.4)$ & $141(29.9)$ & $94(39.3)$ & $0.001^{*}$ \\
\hline Normal & $82(16.6)$ & 69 (26.6) & $6(4.3)$ & $7(7.5)$ & $<0.001^{*}$ \\
\hline Moderately abnormal & $223(45.1)$ & $119(46.0)$ & $76(53.9)$ & $28(29.8)$ & \\
\hline Severely abnormal & $86(17.4)$ & $25(9.7)$ & $37(26.2)$ & $24(25.5)$ & \\
\hline Unknown & 103(20.9) & $46(17.8)$ & $22(15.6)$ & 35 (37.2) & \\
\hline cEEG within $24 \mathrm{~h}^{\#}(\mathrm{n}, \%)$ & $1131(68.2)$ & 707 (74.7) & $310(65.7)$ & 238 (99.6) & $<0.001$ \\
\hline Short & 135 (11.9) & 79 (11.2) & $28(9.0)$ & $28(24.6)$ & $<0.001$ \\
\hline Continuous & $95(8.4)$ & $54(7.6)$ & $24(7.7)$ & $17(14.9)$ & \\
\hline Video & 901 (79.7) & $574(81.2)$ & $258(83.2)$ & $69(60.53)$ & \\
\hline \multicolumn{6}{|l|}{ Diagnosis type } \\
\hline Normal & $172(15.2)$ & $148(20.9)$ & $15(4.8)$ & $9(7.9)$ & $<0.001^{*}$ \\
\hline Abnormal & $959(84.8)$ & $559(79.1)$ & $295(95.2)$ & $105(92.1)$ & \\
\hline \multicolumn{6}{|l|}{ Background } \\
\hline Burst suppression & $123(10.9)$ & $27(3.8)$ & $82(26.5)$ & $14(12.3)$ & $<0.001^{*}$ \\
\hline Continuous (Normal) & $233(20.6)$ & $195(27.6)$ & $21(6.8)$ & $17(14.9)$ & \\
\hline Discontinuous & $620(54.8)$ & $410(58.0)$ & $157(50.7)$ & $53(46.5)$ & \\
\hline Isoelectric / Flat & $103(9.1)$ & $44(6.2)$ & $34(11.0)$ & $25(21.9)$ & \\
\hline Stated unknown & $52(4.6)$ & $31(4.4)$ & $16(5.2)$ & $5(4.4)$ & \\
\hline MRI findings ${ }^{+}(n, \%)$ & 1450 & 831 & 415 & 204 & \\
\hline Hemorrhage & $303(20.9)$ & $166(20.0)$ & $103(24.8)$ & $34(16.7)$ & $0.039^{*}$ \\
\hline Stroke & $107(7.4)$ & $46(5.5)$ & $45(10.8)$ & $16(7.8)$ & $0.003^{*}$ \\
\hline White matter injury & $236(16.3)$ & $98(11.8)$ & $98(23.6)$ & $40(19.6)$ & $<0.001^{*}$ \\
\hline Deep grey matter injury & $284(19.6)$ & $84(10.1)$ & $147(35.4)$ & $53(26.0)$ & $<0.001^{*}$ \\
\hline Cortical injury & $183(12.6)$ & $41(4.4)$ & $115(27.7)$ & $27(13.2)$ & $<0.001^{*}$ \\
\hline Normal & $524(36.1)$ & $400(48.1)$ & $72(17.4)$ & $52(25.5)$ & $<0.001^{*}$ \\
\hline
\end{tabular}

Abbreviations: EEG electroencephalographic, $M R I$ magnetic resonance imaging

\#Based on patients for whom aEEG or cEEG data were available; some patients received both aEEG and full EEG (cEEG); aEEG was not consistently displayed on full EEG; aEEG reflects cerebral function monitor output

${ }^{+}$Based on patients for whom MRI findings were available

* $p$-value $<=0.05$ 
Table 3 Unadjusted AED costs per patient who received AEDs

\begin{tabular}{|c|c|c|c|c|c|c|c|c|}
\hline \multirow[t]{2}{*}{ Category } & \multicolumn{2}{|l|}{ All } & \multicolumn{2}{|c|}{ No Seizure } & \multicolumn{2}{|c|}{ EEG Seizure } & \multicolumn{2}{|c|}{ Clinical Seizure } \\
\hline & $\mathrm{n}$ & median [IQR] cost & $\mathrm{n}$ & median [IQR] cost & $n$ & median [IQR] cost & n & median [IQR] cost \\
\hline All AEDs & 1252 & $\$ 89.90[24.52,265.84]$ & 563 & $\$ 34.13[11.38,100.76]$ & 464 & $\$ 229.39[93.73,511.49]$ & 255 & $\$ 101.15[38.91,209.57]$ \\
\hline Phenobarbital & 739 & $\$ 96.96[35.53,206.04]$ & 107 & $\$ 32.32[13.81,97.86]$ & 437 & $\$ 129.79[59.86,262.06]$ & 195 & $\$ 64.64[24.14,142.23]$ \\
\hline Phenytoin/Fosphenytoin & 118 & $\$ 67.71[15.46,238.53]$ & 3 & $\$ 36.85[29.78,259.06]$ & 97 & $\$ 69.59[15.46,238.53]$ & 18 & $\$ 70.25[25.91,123.94]$ \\
\hline Levetiracetam & 128 & $\$ 197.62[59.25,453.18]$ & 10 & $\$ 69.82[13.84,80.51]$ & 98 & $\$ 240.09[105.10,534.20]$ & 20 & $\$ 53.39[21.48,163.88]$ \\
\hline Benzodiazapines & 1010 & $\$ 34.13[11.38,91.02]$ & 525 & $\$ 26.30[11.37,82.54]$ & 328 & $\$ 45.51[17.33,102.39]$ & 157 & $\$ 34.13[15.39,91.07]$ \\
\hline $\begin{array}{l}\text { Other (oxcarbazepine, gabapentin, } \\
\text { topiramate, valproate) }\end{array}$ & 19 & $\$ 102.94[26.49,354.27]$ & 3 & $\$ 19.18[6.62,56.29]$ & 15 & $\$ 134.22[51.21,379.45]$ & 1 & $\$ 26.49[26.49,26.49]$ \\
\hline
\end{tabular}

Abbreviations: $A E D$ anti-epileptic drug, EEG electroencephalographic, IQR interquartile range

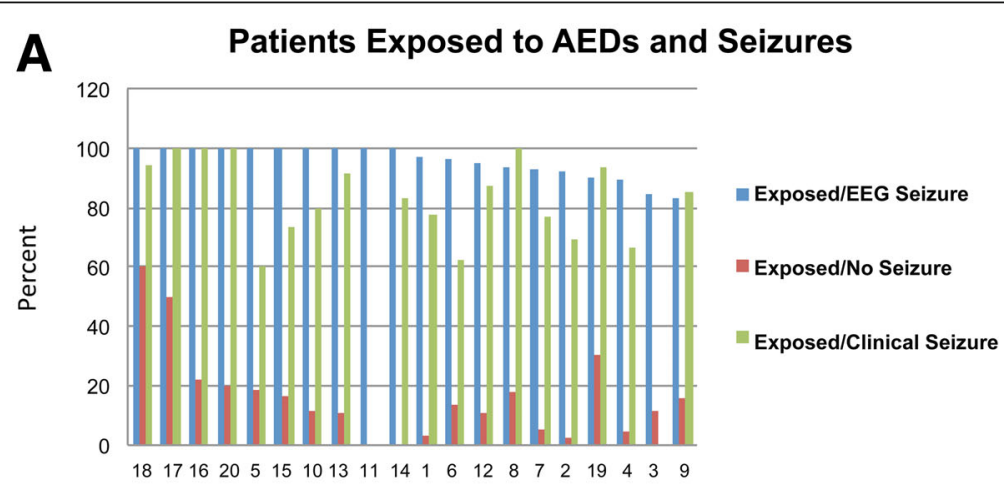

\section{B Patients on Phenobarbital}

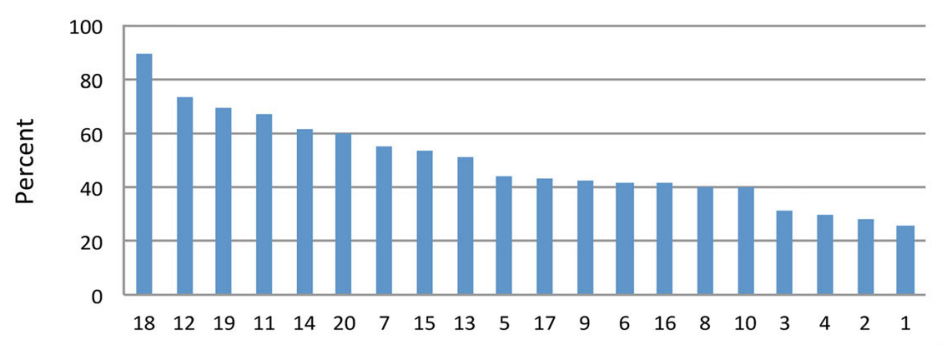

C Patients on Levetiracetam or Phenytoin/Fosphenytoin

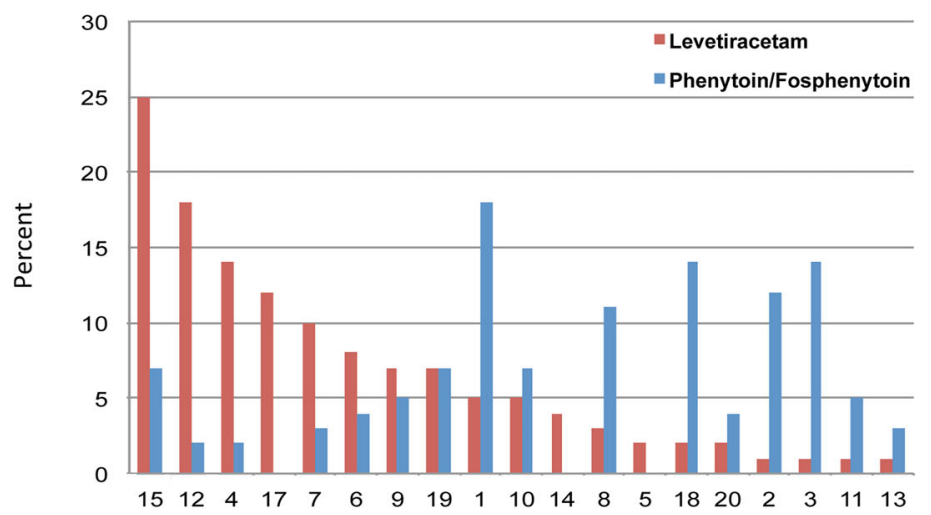

Fig. 2 AED exposure by center. a Rate of exposure of patients to any AEDs with and without electrographic seizures and with clinical seizures by center. $\mathbf{b}$ Exposure to phenobarbital by center. $\mathbf{c}$ Exposure to levetiracetam and phenytoin/fosphenytoin by center 

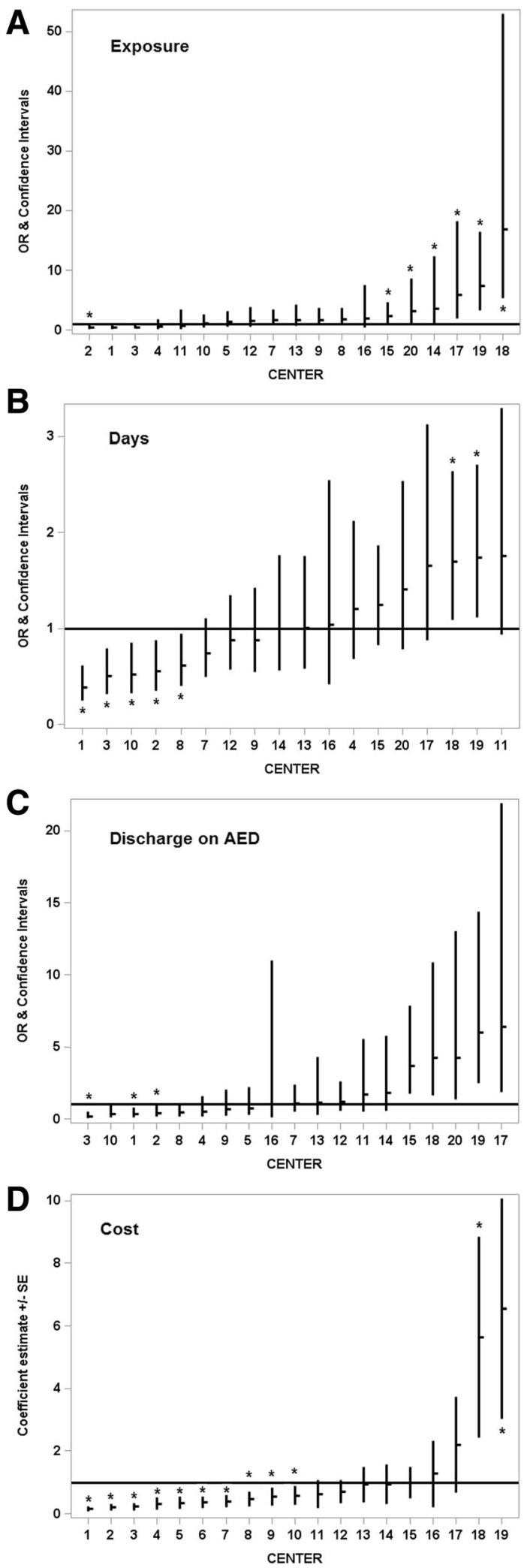

Fig. 3 ICV in AED use and cost. OR (odds ratio). ${ }^{*} p<0.05$. a Any AED exposure by center. b Duration of AED exposure by center. $\mathbf{c}$ Discharge on AED by center. $\mathbf{d}$ Cost of AED use by center

\section{Costs associated with AED use}

The median total unadjusted AED cost per patient who received AEDs was $\$ 89.90$ (IQR \$24.52, \$258.58). Despite similar frequency of use, costs per patient for levetiracetam were over 2.9 times the costs associated with fosphenytoin/phenytoin and twice the cost of phenobarbital (Table 3). In a mixed-effect linear regression model adjusting for gestational age, sex, electrographic seizures, status epilepticus, HIE severity and mortality, AED cost differed significantly across centers $(p<0.001)$, ranging from 0.5 -fold to $>3$-fold (Fig. 3d).

\section{Practice changes over time}

Rates of cEEG use were 54\% at the beginning of the study (2010) versus 64\% for 2012, after publication of American Clinical Neurophysiology Society (ACNS) guidelines for EEGs in neonates [9], and 85\% at the end of the study (2016) (Fig. 4a). Rates of aEEG use were lower than for cEEG throughout the study, highest at $34 \%$ at the beginning of the study with rates of only $19 \%$ at the end of the study (Fig. 4b). Proportion of infants diagnosed with EEG seizures remained relatively stable despite an increase in cEEG use (Fig. 4d). By contrast, proportion of infants with clinical seizures only decreased over time (Fig. 4e). There was a similar decrease in infants who received AEDs when no seizures were detected electrographically, from a peak of $27 \%$ in 2011 to a low of 10\% in 2016 (Fig. 4f). Finally, we looked at rates of individual AEDs by year and observed a decrease in phenobarbital use after 2011, from a peak of $56 \%$ to a low of $38 \%$ in 2015 (Fig. 4g). We also observed that rates of levetiracetam use surpassed rates of fosphenytoin/ phenytoin in 2016 (Fig. 4g).

\section{Discussion}

The purpose of this project was to evaluate the variability that exists across regional NICUs caring for a large burden of neonates with HIE in the US, in order to inform a future QI intervention [41]. In a contemporary cohort of neonates with HIE treated with TH at 20 US regional NICUs, we observed significant ICV in AED utilization. We looked at utilization from a number of different perspectives including selection, any AED exposure, duration of exposure, discharge on AEDs and AED cost as another proxy for utilization. This study of our very large consortium highlighted unwarranted variation [42] in the management of AEDs in HIE, particularly in neonates without electrographic evidence of seizures. This work therefore supports a future QI collaborative across the CHND consortium targeting neonates with HIE who do not have electrographic evidence of seizures. It is important to note that CHND NICUs do not share standardized treatment protocols although all centers have similar levels of care and availability of specialty 

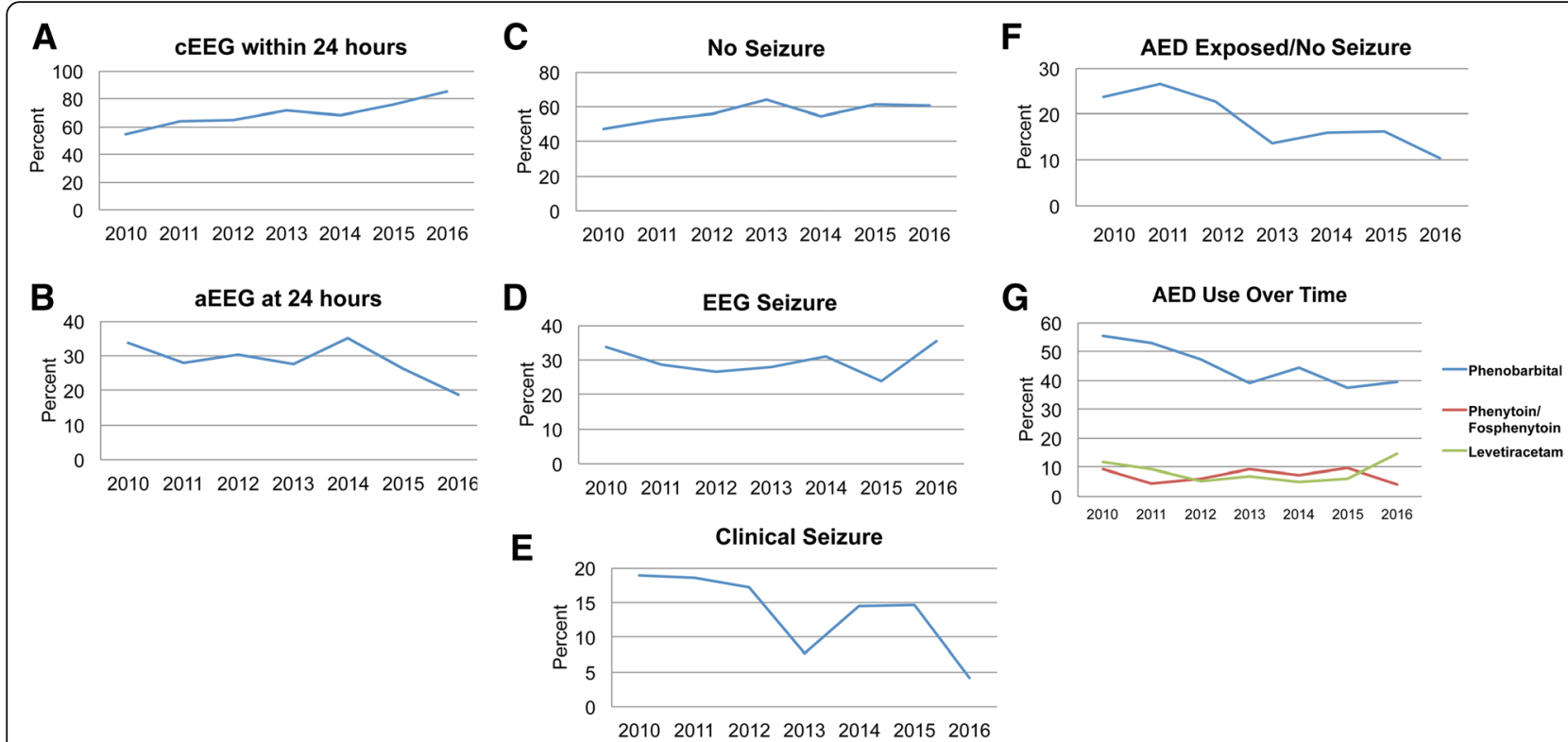

Fig. 4 a CEEG within $24 \mathrm{~h}$ by year. b aEEG at $24 \mathrm{~h}$ by year. c No Seizure by year. $\mathbf{d}$ EEG Seizure by year. e Clinical Seizure by year. $\mathbf{f}$ AED Exposed/ No EEG Seizure by year. g AED Use Over Time by year

services. Although best practices have been designated by the state of California (https://www.dhcs.ca.gov/services/ ccs/Documents/ccsnl061116.pdf), only 2 California sites were involved in this study and more globally accepted guidelines are not available. Nonetheless, all CHND centers involved in this study met recommendations by the American Academy of Pediatrics Committee on Fetus and Newborn for centers that provide TH, including level III or higher NICU care, neurologic consultation, neuromonitoring with aEEG or cEEG, neuroimaging by MRI, systems for monitoring longitudinal neurodevelopmental outcome, training programs and infrastructure including written protocols and monitoring of outcomes as well as outreach to community hospitals [43].

Despite ACNS guidelines for EEGs in neonates [9], significant variability exists in the application of cEEG for seizure detection/monitoring. We did observe a dramatic increase in use of cEEG overall following publication of the guidelines mid-study in 2011. A decrease in use of aEEG use toward the end of the study period may have been related to discontinued sales of the selective head-cooling device in the US. Although $98 \%$ of all neonates in our study received some form of neuro-monitoring (cEEG or aEEG or both), it is possible that our seizure rates are underestimated in those who did not receive monitoring, those who received delayed monitoring or those that received aEEG alone, given the superior sensitivity of cEEG for seizure detection (particularly for seizures that are brief, infrequent or of low amplitude, or not central or parietal [44]). The incidence of seizures detected by EEG in our cohort was $28 \%$, lower than for the CoolCap (61\% detected by aEEG)
[45], TOBY (54\% detected by aEEG) [46] and NICHD hypothermia trials (46\% clinical seizures) [47]. Details regarding exact timing of seizure detection and EEG acquisition in relation to AED administration were not available, although it is known that the majority of seizures in HIE occur in the first $24-48 \mathrm{~h}$ of life $[48,49]$. Status epilepticus rates were lower than expected [2] and may be related to the application of TH to mild HIE cases in real practice. That some clinical seizures occurred in the absence of electrographic seizures might be explained by the following scenarios: clinical movements might not be due to epileptiform activity; seizures noted prior to initiation of cEEG might have spontaneously resolved or resolved following AED given; the threshold to treat clinical seizures during TH might be higher if patients are not on cEEG or aEEG for the entire period of $\mathrm{TH}$ and rewarming; even if they were, cEEG reading might not be immediately available. We observed relatively low rates of clinical seizures but a rate of EEG seizures of nearly $10 \%$ in cases of mild encephalopathy who were cooled. For these cases, we speculate that clinical or EEG seizures might have been noted after initial assignment of severity category without reassignment to the moderate category after seizures were noted. Our data reinforce that $\mathrm{CEEG}$ or aEEG should be obtained in all mild cases of encephalopathy as EEG seizures would indicate that the eligibility for $\mathrm{TH}$ had been met.

Consistent with AED selection in other studies $[8,33$, 34], we observed a similar predominance of phenobarbital use and a higher frequency of levetiracetam compared to phenytoin/fosphenytoin use. We examined 
levetiracetam use by year and found an increase in levetiracetam over fosphenytoin/phenytoin in the final year of the study. The apparent inverse relationship of levetiracetam and fosphenytoin/phenytoin use suggests that preferential use of these second-line medications varies by center practice; alternative explanations include fosphenytoin shortages as well as the development of an intravenous formulation of levetiracetam. AED costs per patient were highest for levetiracetam, 2.9-fold greater than fosphenytoin/phenytoin, and cost considerations may drive AED choice for some providers. On the other hand, levetiracetam may be preferred by some providers because of its association with decreased respiratory depression.

Although previous studies have shown ICV in AED utilization, given that the NICUs in our consortium are all Level IV, we were nonetheless somewhat surprised to find the magnitude of ICV that we observed. One study that included some of the same referral centers, observed similar ICV in continuation of AEDs at discharge for neonatal seizures of all etiologies. After univariable analysis adjusting for electrographically confirmed seizures, status epilepticus, seizures refractory to the initial loading dose of AED and abnormal neurological exam at discharge, only study site and seizure etiology remained significantly associated with discharge on AEDs. With regards to seizures specifically associated with HIE, this study's overall rate of discharge on AEDs was $57 \%$, similar to the $56 \%$ that we observed in cases of HIE with electrographically confirmed seizures. Treatment duration differences were implied in this study but not directly reported [8].

Frequency of AED at discharge was center-dependent in our study as well, suggesting that physician/center practice drives the decision to continue AEDs. In our study, over half of neonates with electrographic seizures and $7 \%$ of neonates without electrographic seizures were discharged on AEDs. Stated otherwise, if a neonate ever received an AED, that neonate had a 1 in 3 chance of being discharged on an AED. This variation is important because, although neonates with HIE, and particularly those with seizures, are at increased risk for later epilepsy [50,51], emerging evidence suggests that discharge on an AED might not be indicated in all neonates with acute seizures after HIE [52]. It is well recognized that prolonged use of most AEDs is associated with neuronal apoptosis and neurodevelopmental delays [26, 53]. This added risk is even less acceptable for neonates who have never demonstrated seizures by EEG. Unlike previous studies, we showed ICV in other measures of AED utilization, including any exposure and duration of exposure and cost.

We were surprised to find that a high proportion of neonates without seizures confirmed by EEG received AEDs, many through discharge. This may partly reflect AED use for clinical seizures not confirmed electrographically, and may occur more frequently when EEG is not immediately obtainable as not all centers have 24/7 EEG technician and neurophysiologist capabilities. High rates of AED use in neonates without electrographic seizures, as high as $60 \%$ at one center, might also reflect attempts at neuroprotection or seizure prophylaxis by some sites. A recent Cochrane Database meta-analysis did not support the use of prophylactic barbiturates for perinatal asphyxia because, although this practice seemed to reduce seizures, it did not reduce mortality or neurodevelopmental impairment [17]. Our data suggests a need to identify sites that use AEDs for neuroprotection or seizure prophylaxis and to stop this practice.

That a small proportion of neonates with electrographic seizures did not receive AEDs during their hospitalization is also surprising. As our data reflects only medications received at CHND hospitals, it is possible that these neonates received AEDs at the referral hospital that were not continued upon admission to the CHND NICU. It is also possible that limited real-time availability of neurophysiologists across centers may be associated with delayed EEG interpretation and reporting, so that some seizures clinically resolved by the time of recognition on EEG would not lead to AED initiation. Finally, although benzodiazepines are often used to treat intractable seizures or status epilepticus, we did not report the use of benzodiazepines that may have been used to treat seizures; given the nature of the registry, we were unable to confirm whether benzodiazepines were given for seizures or for sedation. The use of AEDs without EEG evidence of seizures offers an opportunity for intervention and change in practice(s).

The major strength of our study was the linkage of clinical data with PHIS data which enabled us to evaluate utilization and cost of AEDs over the course of hospitalization in neonates with HIE. Although a previous study used PHIS data to evaluate AED use, its subjects had neonatal seizures due to various etiologies and were hospitalized during an epoch when $\mathrm{TH}$ was not yet standard of care and costs were not evaluated [4]. As $\mathrm{TH}$ has led to centralization of care of neonates with HIE to regional NICUs, describing practice variation in this setting is important. Indeed, not all centers that provide $\mathrm{TH}$ provide related services such as cEEG or aEEG [54]. We capitalized on detailed clinical information from CHND not available from PHIS alone that allowed us to observe that AED use was significantly affected by gestational age, HIE severity, EEG seizures and mortality, in contrast to the previous study [4]. After controlling for these clinical covariates, ICV in AED use for neonates with HIE persisted.

Another major strength of our investigation was that we only studied neonates with HIE, the most common 
etiology of neonatal seizures in the current era, who were cared for at regional NICUs. By contrast, prior studies have compared dissimilar groups such as preterm infants or infants with central nervous system disease [4, 33]. Similarly, prior survey and registry-based studies have evaluated data from various NICUs where availability of neurodiagnostic studies (MRI, EEG, etc.) and child neurology specialists may contribute to variations in care $[7,8]$. Our study included only regional NICUs meeting criteria for participation in the CNHD [27], and thus highlights the true conundrum of unexplained practice variation with regards to AED use in HIE.

Our study has some limitations. Referral biases exist because some neonates may have died prior to referral to the CHND NICU. Coding differences in AED use may exist between centers despite electronic acquisition of data but processes are in place to insure quality [27]. Unfortunately, we were also unable to link EEG findings temporally to AED initiation and discontinuation. Likewise, details regarding timing of seizure detection and EEG performance in relation to discharge were not available, although it is known that the majority of seizures in HIE occur within the first $24-48 \mathrm{~h}$ of life [48, 49]. Developmental outcomes and detailed seizure information is not presently available in CHND. Additionally, given that this study only involved care in regional referral sites, our findings may not be generalizable to community hospitals.

Interestingly, we observed a significantly higher unadjusted rate of seizures in neonates who were selectively head-cooled in contrast with those who received whole body cooling (Table 1). We speculate that delay in obtaining cEEG may result in delay in treatment and a higher rate of seizures at first cEEG. This observation warrants further study given the relatively small number of infants who received selective head cooling, multiple comparisons and unadjusted rates.

\section{Conclusions}

Significant variation exists in AED utilization in neonates with HIE treated with TH within our Children's Hospital regional NICUs. This data indicates a multicenter QI project within the CHND is in order, with the goal of increasing timely neuromonitoring and eliminating exposure to AEDs without proof of EEG seizures. We believe the rate of exposure to AEDs without EEG seizures should approach $0 \%$. Specific practices to be targeted in our initial QI project will include: 1) observation or use of lorazepam for clinical seizure without EEG confirmation, 2) cEEG or aEEG on admission for all neonates transported for $\mathrm{TH}$ (metrics will also include time from admission to placement of cEEG or aEEG), 3) cEEG or aEEG confirmation of seizures prior to phenobarbital, and 4) time from cEEG or aEEG confirmation of seizures to infusion of phenobarbital. Such an effort will improve adherence to evidence-based practices within member hospitals of the CHND.

\section{Abbreviations}

AED: anti-epileptic drug; aEEG: amplitude-integrated EEG; cEEG: continuous electroencephalographic monitoring; CHND: Children's Hospital Neonatal Database; CTC: Clinical Transaction Classification; HIE: hypoxic ischemic encephalopathy; ICV: intercenter variation; MRI: magnetic resonance imaging; PHIS: Pediatric Health Information Systems; QI: quality improvement;

$\mathrm{TH}$ : therapeutic hypothermia

\section{Acknowledgments}

We are indebted to the following institutions that serve the infants and their families; these institutions have also invested in and continue to participate in the CHND. The site sponsors/contributors for the CHND are also included:

1. Children's Healthcare of Atlanta, Atlanta, GA (Francine Dykes, Anthony Piazza).

2. Children's Healthcare of Atlanta at Scottish Rite (Gregory Sysyn).

3. Children's Hospital of Alabama*, Birmingham, AL (Carl Coghill, Allison Black).

4. Le Bonheur Children's Hospital*, Memphis, TN (Ramasubbareddy Dhanireddy).

5. Children's Hospital Boston*, Boston, MA (Anne Hansen, Tanzeema Houssain).

6. Ann \& Robert H. Lurie Children's Hospital of Chicago*, Chicago, IL (Karna Murthy, Gustave Falciglia).

7. Cincinnati Children's Hospital, Cincinnati, OH (Beth Haberman, Breda Poindexter, Amy Nathan, Kristin Nelson, Paul Kingma, Stefanie Riddle, Stephanie Merhar, Heather Kaplan).

8. Nationwide Children's Hospital*, Columbus, OH (Kristina Reber).

9. Children's Medical Center*, Dallas, TX (Rashmin Savani, Luc Brion, Noorjahan Ali).

10. Children's Hospital Colorado*, Aurora, CO (Theresa Grover).

11. Children's Hospital of Michigan*, Detroit, MI (Girija Natarajan).

12. Cook Children's Health Care System*, Fort Worth, TX (Jonathan Nedrelow, Annie Chi, Yvette Johnson).

13. Texas Children's Hospital*, Houston, TX (Gautham Suresh).

14. Riley Children's Hospital, Indianapolis, IN (William Engle, Lora Simpson, Gregory Sokol)

15. Children's Mercy Hospitals \& Clinics*, Kansas City, MO (Eugenia Pallotto).

16. Arkansas Children's Hospital*, Little Rock, AR (Robert Lyle, Becky Rogers).

17. Children's Hospital Los Angeles*, Los Angeles, CA (Steven Chin, Rachel Chapman).

18. American Family Children's Hospital, Madison, WI (Jamie Limjoco, Lori Haack).

19. Children's Hospital \& Research Center Oakland*, Oakland, CA (David Durand, Jeanette Asselin, Art D'Harlingue, Priscilla Joe).

20. The Children's Hospital of Philadelphia*, Philadelphia, PA (Jacquelyn Evans, Michael Padula, David Munson)

21. St. Christopher's Hospital for Children, Philadelphia, PA (Suzanne Touch).

22. Children's Hospital of Pittsburgh of UPMC*, Pittsburgh, PA (Beverly Brozanski)

23. St. Louis Children's Hospital*, St Louis, MO (Tasmin Najaf, Rakesh Rao, Amit Mathur).

24. All Children's Hospital, St. Petersburg, FL (Victor McKay).

25. Rady Children's Hospital*, San Diego, CA (Mark Speziale, Brian Lane, Laural Moyer).

26. Children's National Medical Center*, Washington, DC (Billie Short).

27. Al DuPont Hospital for Children, Wilmington, DE (Kevin Sullivan).

28. Primary Children's Medical Center, Salt Lake City, UT (Con Yee Ling, Shrena Patel).

29. Children's Hospital of Wisconsin*, Milwaukee, WI (Michael Uhing, Ankur Datta).

30. Children's Hospital of Omaha (Lynne Willett, Nicole Birge).

31. Florida Hospital for Children (Rajan Wadhawan).

32. Seattle Children's Hospital, Seattle, WA (Elizabeth Jacobsen-Misbe, Robert DiGeronimo, Zeenia Billimoria).

33. Hospital for Sick Children, Toronto, ON (Kyong-Soon Lee).

34. Children's Hospital Orange County, Los Angeles, CA (Michel Mikhael, Irfan Ahmad).

*centers that contributed data to these analyses.

Funding

No financial assistance was received in support of this study. 


\section{Availability of data and materials}

The datasets generated and/or analyzed during the current study are not publicly available due to privacy reasons but are available from CHND on reasonable request.

\begin{abstract}
Authors' contributions
MD designed the study, interpreted the data, wrote the original and subsequent drafts of the manuscript and is the corresponding author. RR designed the study, interpreted the data, helped write the original draft of the manuscript and edited subsequent drafts. SH designed the study, interpreted the data, helped write the original draft of the manuscript and edited subsequent drafts. IZ helped design the study and performed all statistical analyses. RD designed the study, interpreted the data and edited the manuscript. GN designed the study, interpreted the data and edited the manuscript. JK designed the study, interpreted the data and edited the manuscript. JF designed the study, interpreted the data and edited the manuscript. K-SL designed the study, interpreted the data and edited the manuscript. DS designed the study and interpreted the data. TY designed the study, interpreted the data and edited the manuscript. AM designed the study, interpreted the data and edited the manuscript. ANM led the study design, interpreted the data, helped write the original draft of the manuscript and edited subsequent drafts. All authors read and approved the final manuscript.
\end{abstract}

\section{Ethics approval and consent to participate}

CHND and PHIS data were linked at the patient level using unique identifiers unavailable to investigators. The Institutional Review Board at each participating institution approved participation in CHND and associated research studies. Please see supplemental file for a full list of the Institutional Review Boards of each institution.

\section{Consent for publication}

Not applicable.

\section{Competing interests}

None of the authors have any financial or non-financial conflicts of interest to disclose. Author An Massaro is an Editorial Board Member of BMC Pediatrics.

\section{Publisher's Note}

Springer Nature remains neutral with regard to jurisdictional claims in published maps and institutional affiliations.

\section{Author details}

'Ann \& Robert H. Lurie Children's Hospital of Chicago and Feinberg School of Medicine, Northwestern University, 225 East Chicago Ave, Box 45, Chicago, IL 60611, USA. 'Washington University, St. Louis, MO, USA. ${ }^{3}$ Children's Healthcare of Atlanta, Atlanta, GA, USA. ${ }^{4}$ Children's Hospital Association, Overland Park, KS, USA. ${ }^{5}$ Seattle Children's Hospital/University of Washington, Seattle, WA, USA. ${ }^{6}$ Children's Hospital of Michigan, Detroit, MI, USA. ${ }^{7}$ Penn State Health Children's Hospital, Hershey, PA, USA. ${ }^{8}$ Children's Hospital of Philadelphia, Philadelphia, PA, USA. 'Hospital for Sick Children, Toronto, ON, Canada. ${ }^{10}$ Children's Hospital Colorado, Denver, CO, USA. ${ }^{11}$ University of Pittsburgh School of Medicine, Pittsburgh, PA, USA. ${ }^{12}$ Children's National Health Systems, Washington, DC, USA.

\section{Received: 12 September 2018 Accepted: 20 February 2019}

\section{Published online: 27 February 2019}

\section{References}

1. Wusthoff CJ, Dlugos DJ, Gutierrez-Colina A, Wang A, Cook N, Donnelly M, et al. Electrographic seizures during therapeutic hypothermia for neonatal hypoxic-ischemic encephalopathy. J Child Neurol. 2011;26(6):724-8.

2. Glass HC, Nash KB, Bonifacio SL, Barkovich AJ, Ferriero DM, Sullivan JE, et al. Seizures and magnetic resonance imaging-detected brain injury in newborns cooled for hypoxic-ischemic encephalopathy. J Pediatr. 2011; 159(5):731-5 e1.

3. Massaro AN, Murthy K, Zaniletti I, Cook N, DiGeronimo R, Dizon M, et al. Short-term outcomes after perinatal hypoxic ischemic encephalopathy: a report from the Children's hospitals neonatal consortium HIE focus group. J Perinatol. 2015;35(4):290-6.
4. Blume HK, Garrison MM, Christakis DA. Neonatal seizures: treatment and treatment variability in 31 United States pediatric hospitals. J Child Neurol. 2009;24(2):148-54

5. Murray DM, Boylan GB, Ali I, Ryan CA, Murphy BP, Connolly S. Defining the gap between electrographic seizure burden, clinical expression and staff recognition of neonatal seizures. Arch Dis Child Fetal Neonatal Ed. 2008; 93(3):F187-91.

6. Glass HC, Wusthoff CJ, Shellhaas RA, Tsuchida TN, Bonifacio SL, Cordeiro M, et al. Risk factors for EEG seizures in neonates treated with hypothermia: a multicenter cohort study. Neurology. 2014;82(14):1239-44.

7. Glass HC, Kan J, Bonifacio SL, Ferriero DM. Neonatal seizures: treatment practices among term and preterm infants. Pediatr Neurol. 2012;46(2):111-5.

8. Shellhaas RA, Chang T, Wusthoff CJ, Soul JS, Massey SL, Chu CJ, et al. Treatment duration after acute symptomatic seizures in neonates: a multicenter cohort study. J Pediatr. 2017;181:298-301 e1.

9. Shellhaas RA, Chang T, Tsuchida T, Scher MS, Riviello JJ, Abend NS, et al. The American clinical neurophysiology Society's guideline on continuous electroencephalography monitoring in neonates. J Clin Neurophysiol. 2011; 28(6):611-7.

10. van Rooij LG, Toet MC, van Huffelen AC, Groenendaal F, Laan W, Zecic A, et al. Effect of treatment of subclinical neonatal seizures detected with aEEG: randomized, controlled trial. Pediatrics. 2010;125(2):e358-66.

11. Srinivasakumar P, Zempel J, Trivedi S, Wallendorf M, Rao R, Smith B, et al. Treating EEG seizures in hypoxic ischemic encephalopathy: a randomized controlled trial. Pediatrics. 2015;136(5):e1302-9.

12. Shetty J. Neonatal seizures in hypoxic-ischaemic encephalopathy-risks and benefits of anticonvulsant therapy. Dev Med Child Neurol. 2015;57(Suppl 3):40-3.

13. Wilmshurst JM, Gaillard WD, Vinayan KP, Tsuchida TN, Plouin P, Van Bogaert $P$, et al. Summary of recommendations for the management of infantile seizures: task force report for the ILAE Commission of Pediatrics. Epilepsia. 2015;56(8):1185-97.

14. Painter MJ, Scher MS, Stein AD, Armatti S, Wang Z, Gardiner JC, et al. Phenobarbital compared with phenytoin for the treatment of neonatal seizures. N Engl J Med. 1999;341(7):485-9.

15. Barks JD, Liu YQ, Shangguan Y, Silverstein FS. Phenobarbital augments hypothermic neuroprotection. Pediatr Res. 2010;67(5):532-7.

16. Sarkar S, Barks JD, Bapuraj JR, Bhagat I, Dechert RE, Schumacher RE, et al. Does phenobarbital improve the effectiveness of therapeutic hypothermia in infants with hypoxic-ischemic encephalopathy? J Perinatol. 2012;32(1):15-20.

17. Young L, Berg M, Soll R. Prophylactic barbiturate use for the prevention of morbidity and mortality following perinatal asphyxia. Cochrane Database Syst Rev. 2016;5:CD001240.

18. Glass HC, Poulin C, Shevell Ml. Topiramate for the treatment of neonatal seizures. Pediatr Neurol. 2011;44(6):439-42.

19. Pressler RM, Mangum B. Newly emerging therapies for neonatal seizures. Semin Fetal Neonatal Med. 2013;18(4):216-23.

20. van Rooij LG, Hellstrom-Westas L, de Vries LS. Treatment of neonatal seizures. Semin Fetal Neonatal Med. 2013;18(4):209-15.

21. Pressler RM, Boylan GB, Marlow N, Blennow M, Chiron C, Cross JH, et al. Bumetanide for the treatment of seizures in newborn babies with hypoxic ischaemic encephalopathy (NEMO): an open-label, dose finding, and feasibility phase 1/2 trial. Lancet Neurol. 2015;14(5):469-77.

22. Ikonomidou C, Turski L. Antiepileptic drugs and brain development. Epilepsy Res. 2010;88(1):11-22.

23. Kaushal S, Tamer Z, Opoku F, Forcelli PA. Anticonvulsant drug-induced cell death in the developing white matter of the rodent brain. Epilepsia. 2016; 57(5):727-34.

24. Bittigau P, Sifringer M, Genz K, Reith E, Pospischil D, Govindarajalu S, et al. Antiepileptic drugs and apoptotic neurodegeneration in the developing brain. Proc Natl Acad Sci U S A. 2002;99(23):15089-94.

25. Stefovska VG, Uckermann O, Czuczwar M, Smitka M, Czuczwar P, Kis J, et al. Sedative and anticonvulsant drugs suppress postnatal neurogenesis. Ann Neurol. 2008;64(4):434-45.

26. Sulzbacher S, Farwell JR, Temkin N, Lu AS, Hirtz DG. Late cognitive effects of early treatment with phenobarbital. Clin Pediatr (Phila). 1999;38(7):387-94.

27. Murthy K, Dykes FD, Padula MA, Pallotto EK, Reber KM, Durand DJ, et al. The Children's hospitals neonatal database: an overview of patient complexity, outcomes and variation in care. J Perinatol. 2014;34(8):582-6.

28. Glass HC, Rogers EE, Peloquin S, Bonifacio SL. Interdisciplinary approach to neurocritical care in the intensive care nursery. Semin Pediatr Neurol. 2014; 21(4):241-7. 
29. Harris ML, Malloy KM, Lawson SN, Rose RS, Buss WF, Mietzsch U. Standardized treatment of neonatal status epilepticus improves outcome. J Child Neurol. 2016;31(14):1546-54.

30. Bashir RA, Espinoza L, Vayalthrikkovil S, Buchhalter J, Irvine L, Bello-Espinosa $L$, et al. Implementation of a Neurocritical care program: improved seizure detection and decreased Antiseizure medication at discharge in neonates with hypoxic-ischemic encephalopathy. Pediatr Neurol. 2016;64:38-43.

31. Tracy ET, Mears SE, Smith PB, Danko ME, Diesen DL, Fisher KA, et al. Protocolized approach to the management of congenital diaphragmatic hernia: benefits of reducing variability in care. J Pediatr Surg. 2010;45(6):1343-8

32. Merras-Salmio L, Pakarinen MP. Refined multidisciplinary protocol-based approach to short bowel syndrome improves outcomes. J Pediatr Gastroenterol Nutr. 2015;61(1):24-9.

33. Ahmad KA, Desai SJ, Bennett MM, Ahmad SF, Ng YT, Clark RH, et al. Changing antiepileptic drug use for seizures in US neonatal intensive care units from 2005 to 2014. J Perinatol. 2017;37(3):296-300.

34. Glass HC, Shellhaas RA, Wusthoff CJ, Chang T, Abend NS, Chu CJ, et al. Contemporary profile of seizures in neonates: a prospective cohort study. J Pediatr. 2016;174:98-103 e1.

35. Massaro AN, Murthy K, Zaniletti I, Cook N, DiGeronimo R, Dizon ML, et al. Intercenter cost variation for perinatal hypoxic-ischemic encephalopathy in the era of therapeutic hypothermia. J Pediatr. 2016;173:76-83 e1.

36. Murthy K, Yanowitz TD, DiGeronimo R, Dykes FD, Zaniletti I, Sharma J, et al. Short-term outcomes for preterm infants with surgical necrotizing enterocolitis. J Perinatol. 2014;34(10):736-40.

37. Pasquali SK, Jacobs JP, Shook GJ, O'Brien SM, Hall M, Jacobs ML, et al. Linking clinical registry data with administrative data using indirect identifiers: implementation and validation in the congenital heart surgery population. Am Heart J. 2010;160(6):1099-104.

38. Mongelluzzo J, Mohamad Z, Ten Have TR, Shah SS. Corticosteroids and mortality in children with bacterial meningitis. JAMA. 2008;299(17):2048-55.

39. Keren R, Luan X, Localio R, Hall M, McLeod L, Dai D, et al. Prioritization of comparative effectiveness research topics in hospital pediatrics. Arch Pediatr Adolesc Med. 2012;166(12):1155-64.

40. Shankaran S, Laptook A, Wright LL, Ehrenkranz RA, Donovan EF, Fanaroff AA et al. Whole-body hypothermia for neonatal encephalopathy: animal observations as a basis for a randomized, controlled pilot study in term infants. Pediatrics. 2002;110(2 Pt 1):377-85.

41. Grimshaw JM, Russell IT. Effect of clinical guidelines on medical practice: a systematic review of rigorous evaluations. Lancet. 1993;342(8883):1317-22.

42. Wennberg JE. Forty years of unwarranted variation--and still counting. Health Policy. 2014;114(1):1-2.

43. Committee on F, Newborn, Papile LA, Baley JE, Benitz W, Cummings J, et al. Hypothermia and neonatal encephalopathy. Pediatrics. 2014;133(6):1146-50.

44. Shellhaas RA, Clancy RR. Characterization of neonatal seizures by conventional EEG and single-channel EEG. Clin Neurophysiol. 2007;118(10): 2156-61.

45. Gluckman PD, Wyatt JS, Azzopardi D, Ballard R, Edwards AD, Ferriero DM, et al. Selective head cooling with mild systemic hypothermia after neonatal encephalopathy: multicentre randomised trial. Lancet. 2005;365(9460):663-70.

46. Azzopardi D, Brocklehurst P, Edwards D, Halliday H, Levene M, Thoresen M, et al. The TOBY study. Whole body hypothermia for the treatment of perinatal asphyxial encephalopathy: a randomised controlled trial BMC Pediatr. 2008;8:17.

47. Shankaran S, Laptook AR, Ehrenkranz RA, Tyson JE, McDonald SA, Donovan EF, et al. Whole-body hypothermia for neonates with hypoxic-ischemic encephalopathy. N Engl J Med. 2005:353(15):1574-84.

48. Lynch NE, Stevenson NJ, Livingstone V, Murphy BP, Rennie JM, Boylan GB. The temporal evolution of electrographic seizure burden in neonatal hypoxic ischemic encephalopathy. Epilepsia. 2012;53(3):549-57.

49. Lynch NE, Stevenson NJ, Livingstone V, Mathieson S, Murphy BP, Rennie JM, et al. The temporal characteristics of seizures in neonatal hypoxic ischemic encephalopathy treated with hypothermia. Seizure. 2015;33:60-5.

50. Azzopardi DV, Strohm B, Edwards AD, Dyet L, Halliday HL, Juszczak E, et al. Moderate hypothermia to treat perinatal asphyxial encephalopathy. $\mathrm{N}$ Engl J Med. 2009;361(14):1349-58.

51. Inoue T, Shimizu M, Hamano S, Murakami N, Nagai T, Sakuta R. Epilepsy and west syndrome in neonates with hypoxic-ischemic encephalopathy. Pediatr Int. 2014;56(3):369-72.
52. Fitzgerald MP, Kessler SK, Abend NS. Early discontinuation of antiseizure medications in neonates with hypoxic-ischemic encephalopathy. Epilepsia. 2017;58(6):1047-53.

53. Bittigau $\mathrm{P}$, Sifringer $\mathrm{M}$, Ikonomidou $\mathrm{C}$. Antiepileptic drugs and apoptosis in the developing brain. Ann N Y Acad Sci. 2003;993:103-14 discussion 23-4.

54. Wusthoff CJ, Clark CL, Glass HC, Shimotake TK, Schulman J, Bonifacio SL. Cooling in neonatal hypoxic-ischemic encephalopathy: practices and opinions on minimum standards in the state of California. J Perinatol. 2017.

\section{Ready to submit your research? Choose BMC and benefit from:}

- fast, convenient online submission

- thorough peer review by experienced researchers in your field

- rapid publication on acceptance

- support for research data, including large and complex data types

- gold Open Access which fosters wider collaboration and increased citations

- maximum visibility for your research: over $100 \mathrm{M}$ website views per year

At BMC, research is always in progress.

Learn more biomedcentral.com/submissions 